\title{
Spontaneous formation of suboxidic coordination around Co in ferromagnetic rutile $\mathrm{Ti}_{0.95} \mathrm{Co}_{0.5} \mathrm{O}_{2}$ film
}

\author{
Wen Hu, ${ }^{1}$ Kouichi Hayashi, ${ }^{2}$ a) Tomoteru Fukumura, ${ }^{3}$ Kazuto Akagi, ${ }^{4}$ Masaru Tsukada, ${ }^{4}$ Naohisa Happo, ${ }^{5}$ \\ Shinya Hosokawa, ${ }^{6}$ Kenji Ohwada, ${ }^{7}$ Masamitu Takahasi, ${ }^{7}$ Motohiro Suzuki, $^{8}$ and Masashi Kawasaki ${ }^{9}$ \\ 1) National Synchrotron Light Source II, Brookhaven National Laboratory, Upton, New York 11973, \\ USA \\ 2) Institute for Materials Research, Tohoku University, Sendai 980-8577, Japan \\ 3) Department of Chemistry, The University of Tokyo, Tokyo 113-0033, Japan \\ 4) Advanced Institute for Materials Research, Tohoku University, Sendai 980-8577, \\ Japan \\ ${ }^{5)}$ School of Information Sciences, Hiroshima City University, Hiroshima 731-3194, \\ Japan \\ 6) Department of Physics, Graduate School of Science and Technology, Kumamoto University, Kumamoto 860-8555, \\ Japan \\ 7) Japan Atomic Energy Agency, Hyogo 679-5148, Japan \\ 8) JASRI/SPring-8, Hyogo 679-5198, Japan \\ ${ }^{9)}$ Quantum Phase Electronics Center and Department of Applied Physics, The University of Tokyo, Tokyo 113-8656, \\ Japan
}

(Dated: 8 May 2015)

To evaluate local atomic structures around Co in high temperature diluted ferromagnetic semiconductor Co-doped $\mathrm{TiO}_{2}$, x-ray fluorescence holography and x-ray absorption fine structure experiments were carried out on rutile paramagnetic $\mathrm{Ti}_{0.99} \mathrm{Co}_{0.01} \mathrm{O}_{2}$ and ferromagnetic $\mathrm{Ti}_{0.95} \mathrm{Co}_{0.05} \mathrm{O}_{2}$ films. The Co atoms in the $\mathrm{Ti}_{0.99} \mathrm{Co}_{0.01} \mathrm{O}_{2}$ simply substituted for Ti sites in the rutile structure, whereas a suboxidic arrangement of $\mathrm{CoO}_{2} \mathrm{Ti}_{4}$ formed around $\mathrm{Co}$ in the $\mathrm{Ti}_{0.95} \mathrm{Co}_{0.05} \mathrm{O}_{2}$ films. A theoretical investigation based on a series of first-principles calculations indicated the stability of the aggregated suboxidic clusters in the rutile $\mathrm{TiO}_{2}$, supporting our hypothesis for the formation of suboxidic coordination in the highly Co-doped sample. The suboxidic coordination may be the source of strong exchange interaction, resulting in the high Curie temperature in Co-doped $\mathrm{TiO}_{2}$.

PACS numbers: 61.05.cf, 75.50.Pp, 61.46.Bc

Keywords: Diluted magnetic semiconductor, X-ray fluorescence holography

High-Curie-temperature diluted ferromagnetic semiconductors (high- $T_{C}$ DFSs) are strongly desired for the room-temperature operation of semiconductor spintronic devices. Among them, high- $T_{C}$ DFS oxides have been intensively studied since the discovery of room temperature ferromagnetism in Co-doped $\mathrm{TiO}_{2}$ in $2001^{1,2}$, where the high- $T_{C}$ ferromagnetism was realized by the dilute doping of transition element ions as well as carriers and/or defects ${ }^{3}$. The $T_{C}$ of Co-doped $\mathrm{TiO}_{2}, \sim 600 \mathrm{~K}$, is extraordinarily high considering conventional carrier-mediated ferromagnetism seen in GaMnAs $(\sim 200 \mathrm{~K})$. However, the microscopic origin of the high- $T_{C}$ ferromagnetism has remained a mystery for a long time.

Previously, extrinsic scenario such as Co clustering experimentally observed were proposed as a source of the high- $T_{C}$ ferromagnetism ${ }^{4,5}$. However, recent studies support intrinsic scenario due to the observation of divalent high-spin Co ions without Co metals via soft and hard x-ray photoelectron/absorption spectroscopy ${ }^{6-8}$. While defect-mediated exchange interaction without itinerant carriers was proposed ${ }^{8,9}$, the observation of magnetotransport/magnetooptical properties ${ }^{10,11}$ and the room

a) Electronic mail: khayashi@imr.tohoku.ac.jp temperature ferromagnetism induced by electrostatic and chemical carrier doping ${ }^{12,13}$ indicated the evidence of carrier-mediated ferromagnetism.

The significant amount of oxygen vacancy adjacent to Co is believed to exist in order to satisfy the charge neutrality in Co-doped $\mathrm{TiO}_{2}$ owing to the divalent $\mathrm{Co}$ ion substituting tetravalent $\mathrm{Ti}$ ion, probably resulting in a significant lattice distortion around the Co atoms. Since the microscopic structure could be related with the role of defect in the ferromagnetism, atomic structure particularly around Co ion has to be resolved. Various groups have applied synchrotron-based x-ray spectroscopic techniques.

Using the Co K-edge x-ray absorption fine structure (XAFS), Chambers et al. reported that Co atoms were substituted for $\mathrm{Ti}$ atoms along with the introduction of oxygen vacancies in Co-doped $\mathrm{TiO}_{2}$ anatase ${ }^{14}$. Murakami et al. suggested that both oxidized $\mathrm{Co}^{2+}$ and metallic Co contribute to the ferromagnetism of rutile $\mathrm{TiO}_{2}$ as indicated by XAFS and XPS studies ${ }^{15}$. An x-ray resonant scattering study by Matsumura et al. observed no anomaly between the diffraction intensities at the Co $\mathrm{K}$ edge, suggesting that the Co ions are located at least 0.6 and $1 \AA$ apart from the exact $\mathrm{Ti}$ sites in rutile and anatase $\mathrm{TiO}_{2}$, respectively ${ }^{16}$. Although Griffin Roberts 
et al. predicted that Co atoms occupy preferential interstitial sites in anatase $\mathrm{Co}$-doped $\mathrm{TiO}_{2}{ }^{9}$, the accurate atomic structure around the Co atoms is still unclear experimentally. To investigate the relationship between high- $T_{C}$ ferromagnetism and the local structure, further clarification of the local structure by experimental and theoretical approaches is necessary.

$\mathrm{X}$-ray fluorescence holography $(\mathrm{XFH})$ is a powerful tool for resolving the local structure, enabling the $3 \mathrm{D}$ atomic environment to be visualized around a selected element within a radius of nanometers orders without assuming any prior models ${ }^{17,18}$. To date, the advantageousness of XFH has been demonstrated for the evaluation of local lattice distortions around specified elements ${ }^{19,20}$ and for the detection of nanocluster-like structures ${ }^{21}$. In this work, we experimentally determined local structures around the Co atoms in paramagnetic and ferromagnetic rutile Co-doped $\mathrm{TiO}_{2}$ films by measuring the XFH holograms. In addition, first-principle calculations based on density functional theory were carried out to evaluate the validity of the structure model obtained by the XFH. In the ferromagnetic Co-doped $\mathrm{TiO}_{2}$ film, suboxidic coordination around Co embedded in the rutile structure was observed. This suboxide coordination may generate a ferromagnetic embryo leading to the strong exchange interaction realizing the high- $T_{C}$ in Co-doped $\mathrm{TiO}_{2}$.

In the present work, two rutile $\mathrm{Ti}_{1-x} \mathrm{Co}_{x} \mathrm{O}_{2}$ films with $x=0.01$ and 0.05 were used, where the former and latter are paramagnetic and ferromagnetic, respectively, as discussed in Ref. 22. These films were grown on R$\mathrm{Al}_{2} \mathrm{O}_{3}$ substrates by pulsed laser deposition at an oxygen partial pressure $\mathrm{PO}_{2}$ of $10^{-6}$ Torr. Details of the procedure are described in Ref. 10. The size of each sample is approximately $3 \times 4 \mathrm{~mm}^{2}$ and the surface orientation is that of rutile (101). The XFH experiment was carried out at BL39XU, and XAFS measurements at the $\mathrm{Co}$ and $\mathrm{Ti} \mathrm{K}$ edges were performed at BL22XU of the synchrotron radiation facility SPring-8, Japan. $\mathrm{XFH}$ data were recorded at incident x-ray energies of 8.0 - $12.0 \mathrm{keV}$ in $0.5 \mathrm{keV}$ steps. Using a toroidally bent graphite crystal $^{23}$, Co $\mathrm{K} \alpha(6.93 \mathrm{keV})$ fluorescent $\mathrm{x}$ rays from the samples were analyzed and focused on an avalanche photodiode detector. The Co holograms were measured by scanning the azimuthal angle of the sample $\phi$ in the range of $0^{\circ} \leq \phi \leq 360^{\circ}$ and the incident angle $\theta$ in the range of $0^{\circ} \leq \theta \leq 75^{\circ}$, both in $1^{\circ}$ steps. Details of the setup are given elsewhere. ${ }^{18}$ It took approximately six hours to obtain each hologram. The intensities of the fluorescent $\mathrm{x}$ rays were normalized using the incident $\mathrm{x}$-ray intensities and the hologram oscillation data were obtained by subtracting the background. Figure 1(a) shows the measured hologram pattern of the $\mathrm{Ti}_{0.95} \mathrm{Co}_{0.05} \mathrm{O}_{2}$. The hologram data were symmetrized using the mirrer symmetry of rutile $\mathrm{TiO}_{2}$ (101) (space group: P42/mmm mirror-symmetrized with respect to the dashed circle in Fig. 1(a) and then extended using the rotoreflection symmetry as shown in Fig. 1(b). Such data manipulation is important for providing accurate
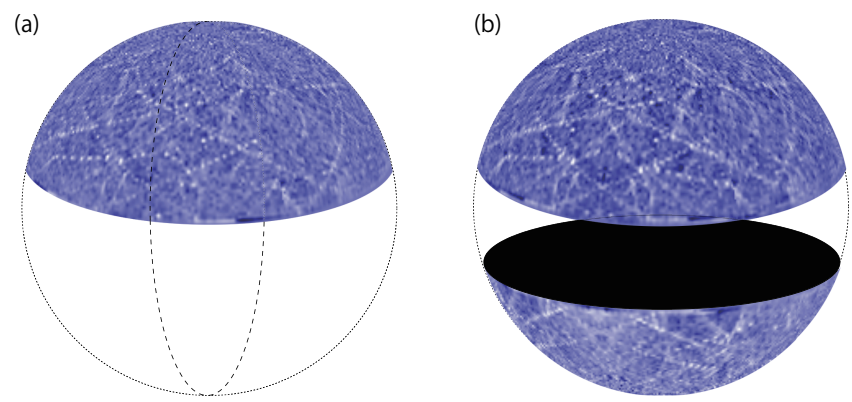

FIG. 1. Co holograms of $\mathrm{Ti}_{0.95} \mathrm{Co}_{0.05} \mathrm{O}_{2}$ film. (a) Raw data and (b) symmetrized data. In (b), the data are mirrorsymmetrized with respect to the dashed circle and then rotoreflection-symmetrized with respect to the (100) plane.

and high-resolution atomic images ${ }^{24}$. The $3 \mathrm{D}$ atomic arrangements around the fluorescent element Co were reconstructed from multiple-energy holograms using Barton's algorithm ${ }^{25}$.

The 3D atomic images of $\mathrm{Ti}_{0.99} \mathrm{Co}_{0.01} \mathrm{O}_{2}$ and $\mathrm{Ti}_{0.95} \mathrm{Co}_{0.05} \mathrm{O}_{2}$ are shown in Figs. 2(a) and (b), respectively. In Fig. 2(a), the reconstructed 3D atomic images are superposed on the ideal $\mathrm{Ti}$ atomic positions of the rutile $\mathrm{TiO}_{2}$ as marked by open circles, while the images of $\mathrm{O}$ atoms are hardly visible owing to their low scattering powers. According to the atomic arrangement of the rutile structure in Fig. 2(c), Fig. 2(a) shows that the Co atoms in the paramagnetic $\mathrm{Ti}_{0.99} \mathrm{Co}_{0.01} \mathrm{O}_{2}$ substituted for $\mathrm{Ti}$ atoms without a large lattice distortion from the rutile structure. In contrast, the $3 \mathrm{D}$ atomic images in Fig. 2(b) are markedly different from the rutile structure. The surrounding atomic arrangement exhibits fourfold symmetry with respect to the normal to the rutile (101) plane (z-axis). The distance between the central Co at the origin and the four nearest-neighboring atomic images at $z=0$ indicated by the arrow is $2.5 \AA$. This distance is similar to the Co-Co or Co-Ti bond length in a Co-Ti alloy ${ }^{26}$, indicating a metal-like in-plane atomic arrangement around the Co. In addition to these four atoms, eight equivalent atomic images are observed at $z$ $=2.5 \AA$. The interatomic distance between the origin and these atomic images is $3.5 \AA$, which is much longer than the aforementioned Co-Co or Co-Ti bond length. Taking into account the very low visibility of $\mathrm{O}$ atoms, we can assume that the $\mathrm{O}$ atoms are located just above and below the Co atom along the $z$-axis as denoted by dashed circles (Fig. 2(d)), corresponding to $\mathrm{CoO}_{2} \mathrm{Ti}_{4}$ coordination as discussed below.

To confirm the validity of our structure models in Figs. 2(c) and (d), we measured the Co K-edge XAFS spectra. Figure 3(a) shows the XAFS spectra of the $\mathrm{Ti}_{0.99} \mathrm{Co}_{0.01} \mathrm{O}_{2}$ and $\mathrm{Ti}_{0.95} \mathrm{Co}_{0.05} \mathrm{O}_{2}$. These dissimilar spectral shapes reflect the different local structures of these samples. To confirm the validity of the $\mathrm{XFH}$ results, we calculated XAFS spectra using the structure models and $\mathrm{FEFF}^{27}$. The XAFS spectrum of the $\mathrm{Ti}_{0.99} \mathrm{Co}_{0.01} \mathrm{O}_{2}$ agrees with that calculated using the ru- 
(a)

(c)
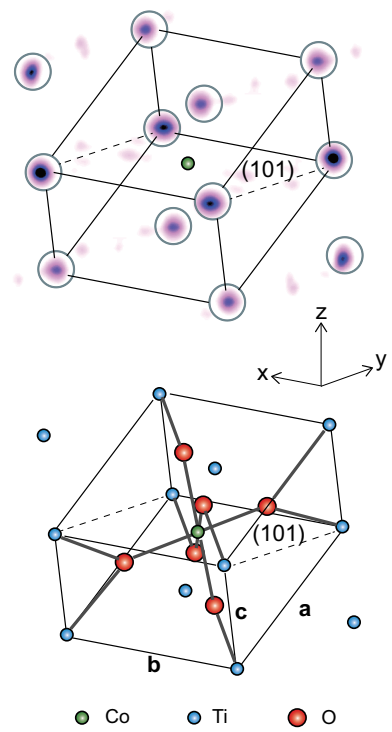

(b)

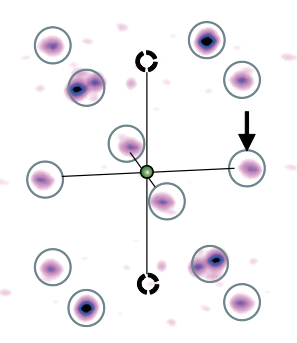

(d)

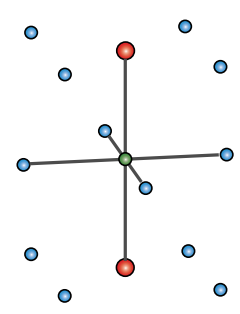

FIG. 2. Reconstructed real-space images around Co in (a) $\mathrm{Ti}_{0.09} \mathrm{Co}_{0.01} \mathrm{O}_{2}$ and (b) $\mathrm{Ti}_{0.95} \mathrm{Co}_{0.05} \mathrm{O}_{2}$ films. (c) and (d) structure models obtained from the images in (a) and (b), respectively.

tile structure in Fig. 2(c), as shown in Fig. 3(a), indicating the validity of the structure obtained by XFH (Fig. $2(\mathrm{c})$ ). The spectrum of the $\mathrm{Ti}_{0.95} \mathrm{Co}_{0.05} \mathrm{O}_{2}$ does not agree with not only that of standard $\mathrm{TiO}_{2}$ but also those of Co metal, CoO, and $\mathrm{CoTiO}_{3}$ powder crystals ${ }^{15}$. Hence, the spectra were calculated using the structure model in Fig. 2(d). Here, because the element (Co or Ti) at the four nearest-neighboring atoms in Fig. 2(b) cannot be identified from the XFH result, both $\mathrm{CoO}_{2} \mathrm{Co}_{4}$ and $\mathrm{CoO}_{2} \mathrm{Ti}_{4}$ structure models were used for the calculation, in which $\mathrm{O}$ atoms were placed in the two dashed circles (Fig. 2(b)). As shown in Fig. 3(a), a difference between these spectra can be seen at the four maxima indicated as a-d. The $\mathrm{CoO}_{2} \mathrm{Ti}_{4}$ structure model more closely reproduces the experimental spectrum of the $\mathrm{Ti}_{0.95} \mathrm{Co}_{0.05} \mathrm{O}_{2}$ film. By fitting the XAFS oscillations calculated using the $\mathrm{CoO}_{2} \mathrm{Ti}_{4}$ model to the experimental oscillations, the interatomic distances of $\mathrm{Co}-\mathrm{Ti}$ and $\mathrm{Co}-\mathrm{O}$ were obtained to be 2.53 and $2.06 \AA$, respectively, in good agreement with the $\mathrm{XFH}$ results given above.

From the results of the XFH and XAFS measurements, we concluded that $\mathrm{CoO}_{2} \mathrm{Ti}_{4}$ clusters were formed in the $\mathrm{Ti}_{0.95} \mathrm{Co}_{0.05} \mathrm{O}_{2}$. These clusters are an intermediate state between the metallic alloy and the oxide. Such a structure is called "suboxide", and is sometimes formed in nonequilibrium states such as $\mathrm{Ru}_{9} \mathrm{O}_{2}{ }^{28}$. Since the Codoped $\mathrm{TiO}_{2}$ film was fabricated in a nonequilibrium process, the formation of a suboxide network is reasonable. In the present case, it is suggested that the large number of oxygen vacancies in the vicinity of the $\mathrm{Co}^{2+}$ resulted in the short bonding length between the $\mathrm{Co}^{2+}$ ions and the neighboring Ti ions. Taking into account the fact

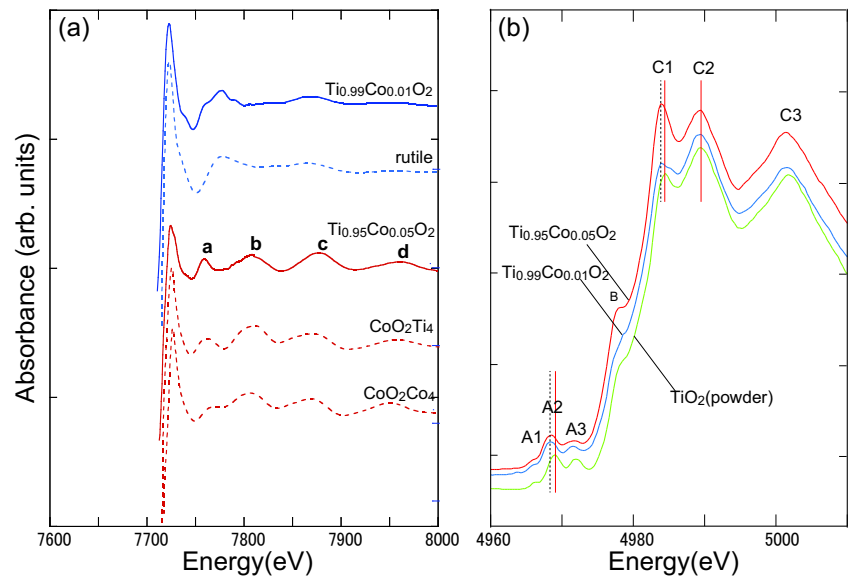

FIG. 3. (color online) (a) Co and (b) Ti K-edge XAFS spectra of $\mathrm{Ti}_{0.99} \mathrm{Co}_{0.01} \mathrm{O}_{2}$ and $\mathrm{Ti}_{0.95} \mathrm{Co}_{0.05} \mathrm{O}_{2}$ films. The solid and dashed lines in (a) indicate experimental and calculated data, respectively.

that metallic precipitation was not observed by $\mathrm{TEM}^{15}$, the size of the suboxide cluster is limited to nanometers order. Since the ferromagnetism in Co-doped $\mathrm{TiO}_{2}$ often occurs in nonequilibrium films, the relation between the high- $T_{C}$ ferromagnetism and the suboxide network is interesting.

We also measured the Ti XAFS of the $\mathrm{Ti}_{0.99} \mathrm{Co}_{0.01} \mathrm{O}_{2}$ and $\mathrm{Ti}_{0.95} \mathrm{Co}_{0.05} \mathrm{O}_{2}$ to examine the effect of suboxide formation on the electronic state of Ti as shown in Fig. 3(b). The x-ray absorption near-edge structure (XANES) spectrum of the rutile $\mathrm{TiO}_{2}$ is characterized by three preedge peaks (A1-3), a shoulder (B), and a split main absorption line $(\mathrm{C} 1,2)$. Compared with the spectrum of standard $\mathrm{TiO}_{2}$, those of the two samples showed a slight shift toward a lower energy by $0.5 \mathrm{eV}$. This feature is noteworthy for the pre-edge peaks corresponding to the $1 s \rightarrow 3 d$ transition, indicating a considerable fraction of $\mathrm{Ti}^{3+}$ ions in both films ${ }^{29}$. In addition, a significant difference between the spectra of the $\mathrm{Ti}_{0.99} \mathrm{Co}_{0.01} \mathrm{O}_{2}$ and $\mathrm{Ti}_{0.95} \mathrm{Co}_{0.05} \mathrm{O}_{2}$ can be seen; the shoulder $\mathrm{B}$ and peak C1 of $\mathrm{Ti}_{0.95} \mathrm{Co}_{0.05} \mathrm{O}_{2}$ appear to be larger than those of $\mathrm{Ti}_{0.99} \mathrm{Co}_{0.01} \mathrm{O}_{2}$. Since the shapes of $\mathrm{B}$ and $\mathrm{C} 1$ are closely related to the atomic arrangement around the absorbing Ti within a radius of $5.5 \AA^{30}$, this result indicates the distortion of the host $\mathrm{TiO}_{2}$ rutile structure possibly due to the formation of suboxide clusters. This feature can also be seen in the Ti K-edge XANES spectrum of an Fe-doped $\mathrm{TiO}_{2}{ }^{31}$.

To evaluate the stability of the suboxide cluster in the host rutile $\mathrm{TiO}_{2}$, we carried out a series of first-principles calculations using the VASP code with the HSE06 hybrid functional for several possible atomic configurations ${ }^{32,33}$. As the first step, to represent an isolated cluster model, the single $\mathrm{CoO}_{2} \mathrm{Ti}_{4}+\left(\mathrm{Ti}_{8}\right)$ cluster depicted in Fig. 2(d) was embedded in the rutile $3 \times 5 \times 4$ supercell structure while keeping the nearest $\mathrm{Ti}$ atoms trivalent. The cluster soon changed into an arrangement similar to that of the 
rutile crystal during the structure optimization process. This result indicates that an isolated $\mathrm{CoO}_{2} \mathrm{Ti}_{4}$ cluster is very unstable, also supporting the absence of the suboxide cluster in the low Co-doped $(x=0.01)$ film. Next, we aligned two $\mathrm{CoO}_{2} \mathrm{Ti}_{4}$ clusters in the rutile $3 \times 3 \times 4$ super cell structure $\left(\mathrm{Co}_{2} \mathrm{O}_{64} \mathrm{Ti}_{34}\right)$, as shown in Fig. 4(a), with an initial configuration where the $\mathrm{O}$ atoms are located above and below the Co atom orthogonal to the cluster alignment, and we obtained the structure in Fig. 4(b) by structure optimization while maintaining the fourfold symmetry of the $\mathrm{CoO}_{2} \mathrm{Ti}_{4}$ clusters around the $z$-axis (see Fig. 2(d)). This structure preserves the original arrangement surrounding the $\mathrm{CoO}_{2} \mathrm{Ti}_{4}$ cluster, reproducing the XFH result of Fig. 2(b). We also calculated the partially and fully relaxed structures for this two-cluster model. The results exhibit large displacements of the four nearest $\mathrm{Ti}$ atoms when the two $\mathrm{O}$ atoms are constrained constraint on the $z$-axis. Moreover, in addition to this displacement, those of the two $\mathrm{O}$ atoms deviate considerably from the original direction when no constrained is imposed. The fourfold symmetry is broken, although the isolated cluster is preserved, as shown in Fig. 4(c). These results imply that the alignment of several clusters may increase the robustness of the local structure against the surrounding rutile structure. It is also suggested that the whole system including the suboxide clusters is an insulator and possesses a finite magnetization regardless the presence or absence of the displacement revealed by the structure optimization. Therefore, the suboxide cluster model is likely to be related to the ferromagnetism.

We also examined a simple substitution model of the $\mathrm{Co}-\mathrm{V}_{O}$ (oxygen vacancy) complex, where the Co substitutes for the Ti site with the neighboring $\mathrm{O}$ vacancy in the rutile structure. The displacement of the Co ion from the exact Ti site was $0.16 \AA$, which is much shorter than the value of $0.6 \AA$ estimated by x-ray resonant scattering ${ }^{16}$. Thus, such a large displacement cannot be attributed to only simple coupling between the substituted Co and the neighboring $\mathrm{O}$ vacancy. Instead, in the case of a large amount of $\mathrm{Co}$ and/or $\mathrm{V}_{O}$, special suboxidic nanoclusters will be formed, generating the irregular Co position with respect to the crystallographic $\mathrm{Ti}$ site in rutile $\mathrm{TiO}_{2}$ structure. Thus, the suboxide cluster model is more plausible than the substitution model with vacancies for explaining the result of the x-ray resonant scattering.

An XAFS spectrum similar to that of the $\mathrm{Ti}_{0.95} \mathrm{Co}_{0.05} \mathrm{O}_{2}$ in Fig. 3(a) was also reported by Kasper et al. ${ }^{34}$, who concluded that their Co-doped $\mathrm{TiO}_{2}$ films contained 10-50\% Co metal because of the similarity of the XANES spectrum to that of Co metal. Considering that Co clusters are not observed usually by TEM and XRD like the report by Murakami et al. ${ }^{15}$, Kasper et al. called this mysterious Co only observed spectroscopically as "hidden Co". However, our experimental result strikingly indicated that the "hidden Co" corresponds to the Co-included suboxide. Some previous XAFS studies suggested the substitution of $\mathrm{Co}$ for the $\mathrm{Ti}$ site with the neighboring oxygen (a)
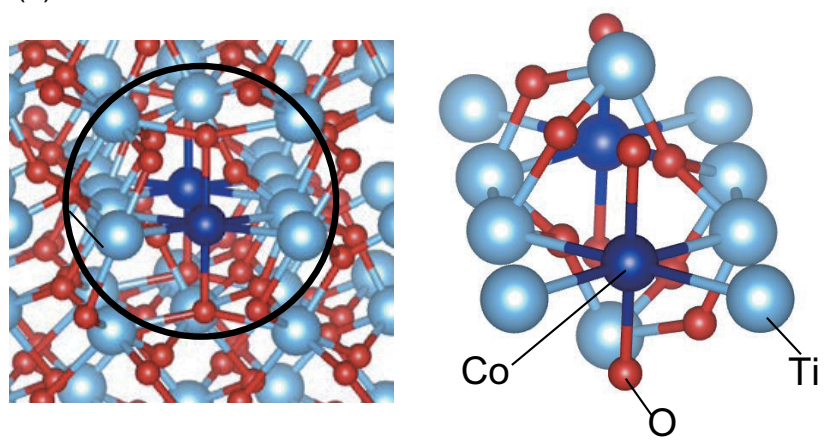

(b)

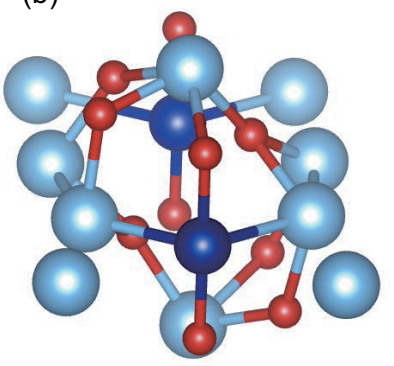

(c)

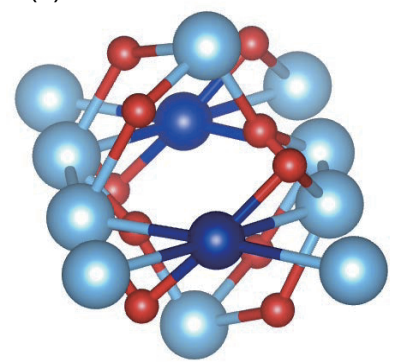

FIG. 4. (color online) Possible structures of the suboxide cluster embedded in rutile $\mathrm{TiO}_{2}$. The structures were obtained by structure optimization based on a first-principles calculation. (a) Two aligned suboxide clusters embedded in rutile $\mathrm{TiO}_{2}$.

(b) Clusters subjected to the constraint condition, where the positions of the oxygen atoms below and above the Co atoms are locked. (c) Fully relaxed clusters.

vacancies $^{14,35}$. However, such simple substitution could not explain the large lattice distortion around Co, as mentioned above.

The Co-included suboxide could be the embryo of the ferromagnetism observed even in the insulating Co-doped $\mathrm{TiO}_{2}$. The carrier mediated exchange interaction between these embryos could induce global ferromagnetism with enhanced magnetization. This scenario could reconcile the long standing two contradictory pictures of this compound: the carrier mediated exchange interaction and defect-mediated one. Some researchers have claimed that the $\mathrm{Co}^{-} \mathrm{V}_{O}$ complex is one of such embryo ${ }^{8,14,35}$, but the suboxidic coordination around Co is more plausible from the results of the present experiment and first principles calculations. If the suboxidic coordination stimulates stronger exchange coupling between the electron carriers and the localized spins than ordinary coordination, the high- $T_{C}$ mechanism in Co-doped $\mathrm{TiO}_{2}$ can be rationalized. This assumption is to be clarified by further theoretical consideration based on first-principles calculations.

In conclusion, the presence of suboxide coordination in a rutile $\mathrm{Ti}_{0.95} \mathrm{Co}_{0.05} \mathrm{O}_{2}$ was revealed by the $\mathrm{XFH}$ and XAFS measurements. Possible atomic configurations of the suboxide network were investigated by first-principles calculation, which justified the reason why the clusters 
appear only at a high Co concentration. Furthermore, the suboxide network might be the source of the strong exchange interaction leading to the high $T_{C}$ in Co-doped $\mathrm{TiO}_{2}$. Note that such a metastable suboxide network can be reproducibly stabilized by a state-of-the-art epitaxial thin film growth technique, as already demonstrated for Co-doped $\mathrm{TiO}_{2}$. Extension of the concept of suboxide coordination to other oxide systems will be an important step forward realizing novel room-temperature DFSs.

\section{ACKNOWLEDGMENTS}

The XFH experiments were performed at BL39XU (Proposal No. 2010A1098). The XAFS experiments were performed at BL22XU (proposal No. 2011A3714). We would also like to acknowledge Japan Society for the Promotion of Science (JSPS) for Grants-in-Aid for Scientific Research (Nos. 25286040 and 26105006).

${ }^{1}$ Y. Matsumoto, M. Murakami, T. Shono, T. Hasegawa, T. Fukumura, M. Kawasaki, P. Ahmet, T. Chikyow, S. Koshihara, and H. Koinuma, Science 291, 854 (2001).

${ }^{2}$ Y. Matsumoto, R. Takahashi, M. Murakami, T. Koida, X. -J. Fan, T. Hasegawa, T. Fukumura, M. Kawasaki, S. Koshihara, and H. Koinuma, pn. J. Appl. Phys., Part 2, 40, L1204 (2001).

${ }^{3}$ S. B. Ogale, Adv. Mater. 22, 3125 (2010).

${ }^{4}$ S. R. Shinde, S. B. Ogale, J. S. Higgins, H. Zheng, A. J. Millis, V. N. Kulkarni, R. Ramesh, R. L. Greene, and T. Venkatesan, Phys. Rev. Lett. 92, 166601 (2004).

${ }^{5}$ J.-Y. Kim, J.-H. Park, B.-G. Park, H.-J. Noh, S.-J. Oh, J. S.Yang, D.-H. Kim, S. D. Bu, T.-W. Noh, H.-J. Lin, H.-H. Hsieh, and C. T. Chen, Phys. Rev. Lett. 90, 017401 (2003).

${ }^{6}$ J. W. Quilty, A. Shibata, J.-Y. Son, K. Takubo, T. Mizokawa, H. Toyasaki, T. Fukumura, and M. Kawasaki, Phys. Rev. Lett. 96, 027202 (2006).

${ }^{7}$ T. Ohtsuki, A. Chainani, R. Eguchi, M. Matsunami, Y. Takata, M. Taguchi, Y. Nishino, K. Tamasaku, M. Yabashi, T. Ishikawa, M. Oura, Y. Senba, H. Ohashi, and S. Shin, Phys. Rev. Lett.106, 047602 (2011).

${ }^{8}$ K. A. Griffin, A. B. Pakhomov, C. M. Wang, S. M. Heald, and K. M. Krishnan, Phys. Rev. Lett. 94, 157204 (2005).

${ }^{9}$ K. Griffin Roberts, M. Varela, S. Rashkeev, S. T. Pantelides, S. J. Pennycook, and K. M. Krishnan, Phys. Rev. B 78, 014409 (2008).

${ }^{10}$ H. Toyosaki, T. Fukumura, Y. Yamada, K. Nakajima, T. Chikyow, T. Hasegawa, H. Koinuma, and M. Kawasaki, Nat. Mater. 3, 221 (2004).

${ }^{11}$ H. Toyosaki, T. Fukumura, Y. Yamada, and M. Kawasaki, Appl. Phys. Lett. 86, 182503 (2005).
${ }^{12}$ Y. Yamada, K. Ueno, T. Fukumura, H. T. Yuan, H. Shimotani, Y. Iwasa, L. Gu, S. Tsukimoto, Y. Ikuhara, and M. Kawasaki, Science 332, 1065 (2011).

${ }^{13}$ Y. Yamada, T. Fukumura, K. Ueno, and M. Kawasaki, Appl. Phys. Lett. 99, 242502 (2011).

${ }^{14}$ S. A. Chambers, S. M. Heald, and T. Droubay, Phys. Rev. B 67, 100401(R) (2003).

${ }^{15}$ M. Murakami, Y. Matsumoto, T. Hasegawa, P. Ahmet, K. Nakajima, T. Chikyow, H. Ofuchi, I. Nakai, and H. Koinuma, J. Appl. Phys. 95, 5330 (2004).

${ }^{16}$ T. Matsumura, D. Okuyama, S. Niioka, H. Ishida, T. Satoh, Y. Murakami, H. Toyosaki, Y. Yamada, T. Fukumura, and M. Kawasaki, Phys. Rev. B 76, 115320 (2007).

${ }^{17}$ M. Tegze and G. Faigel, Nature (London) 380, 49 (1996).

${ }^{18}$ K. Hayashi, N. Happo, S. Hosokawa, W. Hu, and T. Matsushita, J. Phys.: Condens. Matter 24, 093201 (2012).

${ }^{19}$ S. Hosokawa, N. Happo, T. Ozaki, H. Ikemoto, T. Shishido, and K. Hayashi, Phys. Rev. B 87, 094104 (2013).

${ }^{20}$ W. Hu, K. Hayashi, K. Ohwada, J. Chen, N. Happo, S. Hosokawa, M. Takahasi, A. A. Bokov, and Z.-G. Ye, Phys. Rev. B 89, 140103(R) (2014).

${ }^{21}$ W. Hu, K. Hayashi, T. Yamamoto, N. Happo, S. Hosokawa, T. Terai, T. Fukuda, T. Kakeshita, H. L. Xie, T. Q. Xiao, and M. Suzuki, Phys. Rev. B 80, 060202(R) (2009).

${ }^{22}$ T. Fukumura, H. Toyosaki, K. Ueno, M. Nakano, and M. Kawasaki, New J. Phys. 10, 055018 (2008).

${ }^{23}$ T. Sekioka, K. Hayashi, E. Matsubara, Y. Takahashi, T. Hayashi, M. Terasawa, T. Mitamura, A. Iwase, and O. Michikami, J. Synchrotron Rad. 12, 530 (2005).

${ }^{24}$ M. Tegze, G. Faigel, S. Marchesini, M. Belakhovsky, and A. I. Chumakov, Phys. Rev. Lett. 82, 4847 (1999).

${ }^{25}$ J. J. Barton, Phys. Rev. Lett. 67, 3106 (1991).

${ }^{26}$ K. D. Machado, J. C. de Lima, C. E. M. Campos, and T. A. Grandi, Eur. Phys. J. B 37, 421 (2004).

${ }^{27}$ J. J. Rehr and R. C. Albers, Rev. Mod. Phys. 72, 621 (2000).

${ }^{28}$ A. Simon, Coord. Chem. Rev. 163, 253 (1997).

${ }^{29}$ G. A. Waychunas, Am. Mineral. 72, 89 (1987).

${ }^{30}$ F. Farges, G. E. Brown, Jr., and J. J. Rehr, Phys. Rev. B 56, 1809 (1997).

${ }^{31}$ K. Nomura, H. Eba, K. Sakurai, A. Rykov, and T. Hasegawa, Thin Solid Films 515, 8649 (2007).

${ }^{32}$ G. Kresse and J. Furthmuller, Phys. Rev. B 54, 11169 (1996).

${ }^{33}$ J. P. Perdew, J. A. Chevary, S. H. Vosko, K. A. Jackson, M. R. Pederson, D. J. Singh, and C. Fiolhais, Phys. Rev. B 46, 6671 (1992).

${ }^{34}$ T. C. Kaspar, T. Droubay, C. M. Wang, S. M. Heald, A. S. Lee, and S. A. Chambers, J. Appl. Phys. 97, 073511 (2005).

${ }^{35}$ W. Yan, Z. Sun, Z. Pan, Q. Liu, T. Yao, Z. Wu, C. Song, F. Zeng, Y. Xie, T. Hu, and S. Wei, Appl. Phys. Lett. 94, 042508 (2009). 